\title{
User Grouping Based on Large Scale Channel Information in Multi-cell Downlink Cooperative Multi-antenna Systems
}

\author{
Can Sun, Chenyang Yang \\ Beihang University, Beijing, China \\ Email: saga@ee.buaa.edu.cn \\ cyyang@buaa.edu.cn
}

\author{
Yonghui Li, Branka Vucetic \\ University of Sydney, Sydney, Australia \\ Email: 1yh@ee.usyd.edu.au \\ branka@ee.usyd.edu.au
}

\begin{abstract}
In this paper, we study low feedback user grouping in downlink cooperative transmission multi-antenna systems. Users in the same group are served on the same frequency and time resource by multiple cooperative BSs using the zeroforcing precoder. We first derive the user's average received signal to interference and noise ratio (SINR). It shows that each user's SINR is an increasing function of the large scale signal to interference ratio (SIR) of users in neighbor cells, which can be obtained using the large scale channel information. We then propose a novel fair user grouping algorithm based on the large scale SIR. Simulation results show that our algorithm achieves better cell edge throughput compared with existing methods with minor reduction of cell throughput.
\end{abstract}

\section{INTRODUCTION}

Base station (BS) cooperative transmission is a promising strategy to provide high spectral efficiency for next generation cellular systems by avoiding the inter-cell interference [1]. For downlink cooperative transmission, linear precoding such as zero-forcing (ZF) precoder associated with power allocation and user scheduling is a feasible solution which can trade off the performance and complexity [2]-[4].

When instantaneous channel state information (CSI) from all users in the coordinated cells are known by the BSs, the group of users who are proper to share the same frequency and time resource can be selected. However, the overhead to acquire the CSI is huge even in single-cell multi-user systems with a moderate number of users. Another critical issue in scheduling is fairness, especially in cellular systems where the users are scattered across the cell [5], [6]. Although many existing fair scheduling methods can be applied for BS cooperative transmission systems as well, their performance will become different [5]-[7] and they can not operate with only large scale CSI.

Many low feedback user schedulers have been proposed in single-cell systems, e.g., [8], [9]. Feedback requirement in schedulers grows linearly with the coordinated cells in downlink cooperative multi-antenna systems, which even exceeds its resulting gain. In BS cooperative transmission systems, the channels exhibit peculiar characteristics such as asymmetric, i.e., the average signal power received by each user from different BSs are different. By taking advantage of this feature,
[10] proposed a channel norm based scheduler (NUS) given a ZF precoder, which uses opportunistic Round-robin scheduling to provide short term fairness while achieve maximal sum rate in each time slot. To achieve BS cooperation under constrained backhaul, [11] presented an isolation based grouping (IBG) method using large scale CSI, where in general the cell center users are divided into a group and the cell edge users into another group. [3], [4] also proposed user scheduling methods using the large scale CSI. Since the authors aimed at maximizing the sum rate of the system, only cell center user will be selected, while the cell edge users will never be served.

In this paper, we study fair user group using large scale CSI by exploiting the asymmetric channel characteristics in downlink cooperative transmission systems.

We first analyze the average received signal to interference and noise ratio (SINR) in a downlink cooperative transmission system using ZF precoder. It shows that the average received SINR of any user is an increasing function of the large scale signal to interference ratio (SIR) of the users in neighbor cells. We then propose a user grouping algorithm based on the large scale SIR considering the fairness among users, called "strongweak" grouping. We address the fairness issue in a novel way by exploiting the unique feature of channels inherent in the cooperative transmission systems. Different from existing fair scheduling methods that divide time or frequency resources among users more evenly [5], [6], our method can improve the throughput of the cell edge users by "borrowing" the power resource from the cell center users. The simulation results show that our algorithm achieves better cell edge throughput than existing methods with minor cell throughput reduction.

\section{SYSTEM MODEL}

We consider a downlink cooperative transmission system consisting of $N_{B}$ BSs. Each BS is equipped with $N_{T}$ antennas, and serves $N_{T}$ single-antenna users simultaneously. The cooperative BSs transmit to all the $N_{U}=N_{T} N_{B}$ users using the ZF precoder. We assume that all the BSs are connected via high speed backbone networks so that all the channel and data information can be shared among these BSs. The received 
signals at $N_{U}$ users can be expressed as

$$
\mathbf{y}=\mathbf{H W} \mathbf{x}+\mathbf{n},
$$

where $\mathbf{x}$ and $\mathbf{n}$ denote the desired signal and the noise vector of all $N_{U}$ users respectively subjecting to complex Gaussian distribution i.e., $\mathcal{C N}(0, \mathbf{I})$ and $\mathcal{C N}\left(0, \sigma_{n}^{2} \mathbf{I}\right)$, I is an identity matrix. $\mathbf{W}$ is the cooperative $\mathrm{ZF}$ precoding matrix. The $i j$ th element of the composite channel matrix $\mathbf{H}$ is $\alpha_{i j} h_{i j}$, where $\alpha_{i j}$ and $h_{i j}$ respectively denote the large scale and small scale channel fading gains from the $j$ th transmit antenna to the $i$ th user. All $h_{i j}$ s are assumed to be independent identically distributed (i.i.d.) complex Gaussian random variables with zero mean and unit variance. $\alpha_{i j}$ is composed of path loss and shadowing.

Accurate system and channel model are critical for performance analysis and system design, while a realistic multicell system model will render analytical treatment extremely hard or even impossible. As a result, many analysis for multicell system such as [3], [4], [12] are based on the "Wyner model". We also consider this model for analysis, where all $N_{B}$ cooperative BSs locate uniformly along a circle and each user can only be "seen" by two closest BSs. Then the large scale channels of the $i$ th user are modeled as,

$$
\alpha_{i j}=\left\{\begin{array}{l}
1, j_{c}=i_{c}, \\
\alpha_{i}, j_{c}=\left[i_{c}\right]_{N_{B}}+1,\left(0<\alpha_{i}<1\right) \\
0, \text { others } .
\end{array}\right.
$$

where $i_{c}$ and $j_{c}$ are the respective indices of the cells in which the $i$ th user and the $j$ th transmitter antenna are located, and $[i]_{N}$ denotes $i$ modulo $N$. We make a little modification on the Wyner Model here that each user has different $\alpha_{i}$, i.e., each user has different large scale SIR $1 / \alpha_{i}^{2}$. Smaller value of $\alpha_{i}$ means the $i$ th user suffers lower interference from neighbor cells. If $\alpha_{i}$ approaches 0 , then the $i$ th user is a cell center user that almost do not interfered by neighbor BSs.

We consider per-user-power-constraint (PUPC) and each user has identical power constraint. While per-BS-powerconstraint (PBPC) [13], [14] is more proper in the context, PUPC leads to tractable analysis [15]. Moreover, it is worth to note that in multi-carrier systems, when we perform PUPC on each sub-carrier, the PBPC over all carriers will be automatically satisfied due to the averaging over multiple carriers.

\section{USER GROUPING USING LARGE SCALE CHANNEL}

Without loss of generality, we take the 1st user as an example for our analysis. The received signal of the 1st user can be written as,

$$
y_{1}=\mathbf{h}_{1} \mathbf{w}_{1}^{T} x_{1}+\sum_{i=2}^{N_{U}} \mathbf{h}_{1} \mathbf{w}_{i}^{T} x_{i}+n_{1}
$$

where $\mathbf{h}_{i}$ and $\mathbf{w}_{i}$ denote the $i$ th row of $\mathbf{H}$ and $\mathbf{W}^{T} .(\cdot)^{T}$ denotes the transpose of a vector or a matrix. The first, second and the third term are the desired signal, the interference and the noise, respectively. The second term is zero since we use a ZF precoder.
Denote $\mathbf{w}_{1}=\left(w_{11}, w_{12}, \ldots, w_{1 N_{B} N_{T}}\right)$ as the ZF precoder vector for the 1st user. Then it satisfies the following orthogonality condition and PUPC constraint,

$$
\left\{\begin{array}{l}
\overline{\mathbf{H}}_{1} \mathbf{w}_{1}^{T}=\mathbf{0} \\
\left\|\mathbf{w}_{1}\right\|^{2}=1
\end{array}\right.
$$

where $\overline{\mathbf{H}}_{1}=\left[\mathbf{h}_{2}^{T}, \mathbf{h}_{3}^{T}, \cdots, \mathbf{h}_{N_{U}}^{T}\right]^{T}$ is the channel matrix for all users other than the 1 st user. $\|\cdot\|$ is the norm of a vector or a matrix.

Since $\mathbf{w}_{1}$ is a function of $\overline{\mathbf{H}}_{1}$ and $\overline{\mathbf{H}}_{1}$ does not include $\mathbf{h}_{1}, \mathbf{w}_{1}$ and $\mathbf{h}_{1}$ are statistically independent. Then the average received SINR of the 1st user can be written as,

$$
\begin{aligned}
\bar{\gamma}_{1} & =E\left(\left\|\mathbf{h}_{1} \mathbf{w}_{1}^{T}\right\|^{2}\right) / \sigma_{n}^{2} \\
& =E\left(\sum_{i B}^{N_{B} N_{T}} \alpha_{1 i}^{2}\left|h_{1 i}\right|^{2}\left|w_{1 i}\right|^{2}\right) / \sigma_{n}^{2} \\
& =E\left(\sum_{i=1}^{N_{B} N_{T}} \alpha_{1 i}^{2}\left|w_{1 i}\right|^{2}\right) / \sigma_{n}^{2}
\end{aligned}
$$

where $E(\cdot)$ denotes the expectation of a random variable.

In single-cell systems, all transmitter antennas locate at the same BS. The large scale channel gain from these antennas to the 1 st user will be identical, i.e., $\alpha_{11}=\cdots=\alpha_{1 N_{B} N_{T}}$. Then the average received SINR of the 1st user becomes

$$
\bar{\gamma}_{1 s}=\alpha_{11}^{2} E\left(\sum_{i=1}^{N_{B} N_{T}}\left|w_{1 i}\right|^{2}\right) / \sigma_{n}^{2}=\alpha_{11}^{2} / \sigma_{n}^{2}
$$

which does not depend on the channel gains of other users.

In multi-cell systems, the $N_{T} N_{B}$ transmit antennas locate at different BSs. The large scale channel gains are no longer identical. From (5), we can find that any user's average received SINR is a function of other user' large scale channels. This implies that exploiting large scale channel information for selecting users to be served together can improve the performance of the system.

In the following, we first analyze the 1st user's average received SINR in single-antenna BS systems, then we extend the result to multi-antenna BS scenarios. Based on the analysis, we propose a user grouping algorithm to improve the users's average received SINR.

\section{A. Single-antenna $B S$}

In this subsection, we assume that each BS has only one antenna thus each BS can only serve one user. According to the Wyner model, channel matrix can be expressed as

$$
\mathbf{H}=\left(\begin{array}{cccc}
h_{11} & \alpha_{1} h_{12} & \cdots & 0 \\
0 & h_{22} & \ddots & \vdots \\
\vdots & \vdots & \ddots & \alpha_{N_{B}-1} h_{N_{B}-1 N_{B}} \\
\alpha_{N_{B}} h_{N_{B} 1} & 0 & \cdots & h_{N_{B} N_{B}}
\end{array}\right)
$$

Substituting (7) into (4) and using the concept of adjoint matrix [16], we can obtain each element of $\mathbf{w}_{1}$ as

$$
w_{1 k}=D_{1 k} / \sqrt{\left|D_{11}\right|^{2}+\cdots+\left|D_{1 N_{B}}\right|^{2}}, 1 \leq k \leq N_{B}
$$

where $D_{1 k} \mathrm{~s}$ are the respective cofactors of elements in the first row of $\mathbf{H}$, which are $D_{11}=\prod_{i=2}^{N_{B}} h_{i i}, D_{1 k}=$ $(-1)^{k+1} \prod_{i=2}^{k-1} h_{i i} \prod_{i=k}^{N_{B}} \alpha_{i} h_{i[i+1]_{N_{B}}}, k \geq 2$. 
Substituting (7) and (8) into (5), we can obtain the average received SINR of the 1st user as

$$
\begin{aligned}
\bar{\gamma}_{1} & =E\left(\left|w_{11}\right|^{2}+\alpha_{1}^{2}\left|w_{12}\right|^{2}\right) / \sigma_{n}^{2} \\
& =E\left[\left(\left|D_{11}\right|^{2}+\alpha_{1}^{2}\left|D_{12}\right|^{2}\right) /\left(\sigma_{n}^{2} \sum_{i=1}^{N_{B}}\left|D_{1 i}\right|^{2}\right]\right)
\end{aligned}
$$

Since

$$
\frac{\partial\left|D_{1 k}\right|^{2}}{\partial \alpha_{N_{B}}}= \begin{cases}0 & , k=1 \\ 2\left|D_{1 k}\right|^{2} / \alpha_{N_{B}}, & k \geq 2\end{cases}
$$

we have,

$$
\begin{aligned}
\frac{\partial \bar{\gamma}_{1}}{\partial \alpha_{N_{B}}}= & \frac{1}{\sigma_{n}^{2}} E\left\{\left[\alpha_{1}^{2} \frac{\partial\left|D_{12}\right|^{2}}{\partial \alpha_{N_{B}}} \sum_{i=1}^{N_{B}}\left|D_{1 i}\right|^{2}-\left(\left|D_{11}\right|^{2}\right.\right.\right. \\
& \left.\left.\left.+\alpha_{1}^{2}\left|D_{12}\right|^{2}\right) \sum_{i=1}^{N_{B}} \frac{\partial\left|D_{1 i}\right|^{2}}{\partial \alpha_{N_{B}}}\right] /\left(\sum_{i=1}^{N_{B}}\left|D_{1 i}\right|^{2}\right)^{2}\right\} \\
= & \frac{2}{\alpha_{N_{B}} \sigma_{n}^{2}} E\left\{\left[\alpha_{1}^{2}\left|D_{12}\right|^{2} \sum_{i=1}^{N_{B}}\left|D_{1 i}\right|^{2}-\left(\left|D_{11}\right|^{2}\right.\right.\right. \\
& \left.\left.\left.+\alpha_{1}^{2}\left|D_{12}\right|^{2}\right) \sum_{i=1}^{N_{B}}\left|D_{1 i}\right|^{2}\right] /\left(\sum_{i=1}^{N_{B}}\left|D_{1 i}\right|^{2}\right)^{2}\right\} \\
= & -\frac{2}{\sigma_{n}^{2} \alpha_{N_{B}}} E\left\{\left|D_{11}\right|^{2} / \sum_{i=1}^{N_{B}}\left|D_{1 i}\right|^{2}\right\}<0
\end{aligned}
$$

This indicates that the average received SINR $\bar{\gamma}_{1}$ of the 1 st user is a descending function of $\alpha_{N_{B}}$. According to the assumption in Wyner model, the large scale SIR of the user in the $N_{B}$ th cell is $1 / \alpha_{N_{B}}^{2}$. Therefore, $\bar{\gamma}_{1}$ is an increasing function of the large scale SIR of the user in the $N_{B}$ th cell.

Here, we give an intuitive interpretation. In the Wyner model, the $N_{B}$ th cell is the only cell that can be interfered by the 1st BS. If the user in that cell has strong large scale SIR, it implies that the 1st BS generates minor interference to the $N_{B}$ th cell, and 1st BS can serve the 1st user with maximum power under PUPC constraint to achieve high average received SINR. On the other hand, if user in the $N_{B}$ th cell has weak large scale SIR, the 1st BS can no longer transmit with high power. Otherwise it will degrade the performance of the user in the $N_{B}$ th cell.

In realistic cellular systems, one BS will cause interference to all neighbor cells. We will show through simulations in next section that the conclusion drawn from Wyner model also applies for practical systems, where any user's average SINR is an increasing function of the large scale SIRs of users in all the neighbor cells.

\section{B. Multiple-antenna $B S$}

Now we consider that each BS has more than one antennas, i.e., $N_{T}>1$. The channel matrix is given by

$$
\mathbf{H}=\left(\begin{array}{cccc}
\mathbf{H}_{11} & \mathbf{H}_{12} & \cdots & 0 \\
0 & \mathbf{H}_{22} & \ddots & \vdots \\
\vdots & \vdots & \ddots & \mathbf{H}_{N_{B}-1} N_{B} \\
\mathbf{H}_{N_{B} 1} & 0 & \cdots & \mathbf{H}_{N_{B} N_{B}}
\end{array}\right)
$$

where the $i j$ th submatrix $\mathbf{H}_{i j}$ denotes the channel matrix between the users in the $i$ th cell and the $j$ th BS, whose $m n$th element is $\alpha_{(i-1) N_{T}+m} h_{(i-1) N_{T}+m}(j-1) N_{T}+n$.

Substituting the first row of the channel matrix into (5), then the average received SINR of the 1st user is,

$$
\bar{\gamma}_{1}=E\left[\sum_{i=1}^{N_{T}}\left|w_{1 i}\right|^{2}+\alpha_{1}^{2} \sum_{i=N_{T}+1}^{2 N_{T}}\left|w_{1 i}\right|^{2}\right] / \sigma_{n}^{2}
$$

It is hard to get an explicit expression for $\bar{\gamma}_{1}$ in this scenario, we consider a special case where all the users in the $N_{B}$ th cell have strong large scale SIR and we use simulation to validate our conclusion in next section. In this case, the large scale channel coefficients from all the users in the $N_{B}$ th cell to the 1st BS are closed to 0, then the sub-matrix $\mathbf{H}_{N_{B} 1}$ in (12) will approximately be a zero matrix. Using the $\mathrm{ZF}$ constraint and PUPC condition in (4), we can get

$$
\sum_{i=(k-1) N_{T}+1}^{k N_{T}}\left|w_{1 i}\right|^{2} \approx \begin{cases}1, & k=1, \\ 0, & 2 \leq k \leq N_{B} .\end{cases}
$$

Substituting (14) into (13), we can get the value of $\bar{\gamma}_{1}$ under this special case as $1 / \sigma_{n}^{2}$, which is a upper bound of $\bar{\gamma}_{1}$. Proof of the upper bound is as follows, where $\alpha_{1}<1$ and PUPC constraint $\sum_{i=1}^{N_{B} N_{T}}\left|w_{1 i}\right|^{2}=1$ are used.

$$
\begin{aligned}
\bar{\gamma}_{1} & =E\left[\sum_{i=1}^{N_{T}}\left|w_{1 i}\right|^{2}+\alpha_{1}^{2} \sum_{i=N_{T}+1}^{2 N_{T}}\left|w_{1 i}\right|^{2}\right] / \sigma_{n}^{2} \\
& <E\left[\sum_{i=1}^{N_{T}}\left|w_{1 i}\right|^{2}+\sum_{i=N_{T}+1}^{2 N_{T}}\left|w_{1 i}\right|^{2}\right] / \sigma_{n}^{2} \\
& <1 / \sigma_{n}^{2}
\end{aligned}
$$

It indicates that $\bar{\gamma}_{1}$ achieves its maximum value when all users in the $N_{B}$ th cell have strong large scale SIR. Therefore, we can draw the same conclusion in multi-antenna BS scenario as in single antenna BS scenario, i.e., the performance of each user can be improved by grouping it with users that have strong large scale SIR in neighbor cells.

\section{User grouping based on large scale SIR}

Traditional channel-aware scheduling methods need instantaneous CSI [2]. To reduce the feedback, we propose a user grouping method only using large scale CSI based on the precious analysis.

We divide all the users in all the $N_{B}$ cells into a number of user groups each consisting of $N_{U}$ users. Although scheduling among the user groups provides the flexibility to further improve performance, we only focus on how to divide users into groups exploiting the asymmetry channel feature.

In the following, we call the users with strong large scale SIR as "strong" users, those with weak large scale SIR as "weak" users. According to pervious analysis, we know that the performance of any user can be improved by grouping it with "strong" users in its neighbor cells. This implies that the grouping demands of all users are conflict among each other. To achieve better performance, every user desires to be grouped with "strong" users in other cells. The question is whose demand should be given a higher priority.

Since the users located at cell center always operate at high SINR, their performance will degrade little if they "lend" some power to the "weak" users that usually located at cell edge. 
Meanwhile, the performance of those cell edge users can be improved significantly if they are provided more power. We propose a heuristic user grouping method which give "weak" users higher priority to be grouped with "strong" users in neighbor cells. Eventually, "strong" users in some cells and "weak" users in other cells will be grouped togeter, so we call the method as "strong-weak" grouping (SWG) method.

Now let us consider the fairness among the BSs. To obtain higher cell edge throughput, every BS wants to group its "weak" users with "strong" users in all its neighbor cells. It is impossible to fulfill the demands of all the cells as well. To solve this problem, we give each BS an index and divide all BSs into two parts, BSs with odd index (the odd BSs) and BSs with even index (the even BSs). We group the "weak" users of odd BSs with the "strong" users of even BSs, while group the "strong" users of odd BSs with the "weak" users of even BSs. Then every cell has a chance to group its "weak" users with "strong" users in two or three neighbor cells.

The procedure of our SWG algorithm is as follows.

1) Every user calculates its large scale SIR and feeds it back to its own serving BS.

2) Every BS sorts the users by large scale SIR.

3) Give each BS an index and divide all BSs into 2 parts, the odd BSs and the even BSs.

4) Group the "strongest" $N_{t}$ users in each odd BS and the "weakest" $N_{t}$ users in each even BS together, group the second "strongest" $N_{t}$ users in each odd BS and the second "weakest" $N_{t}$ users in each even BS together, $\ldots$, until all the users are grouped.

The IBG algorithm presented in [11] is actually a "strongstrong" grouping method. It improves the performance of cell center users but not cell edge users. Our SWG algorithm has the same overhead as IBG, which needs much less amount of feedback than conventional channel aware schedulers.

\section{Simulation Results}

In this section, we first validate our conclusions by simulation using a realistic multi-cell model. Then we evaluate the throughput performance of our SWG algorithm.

We consider a cooperating cluster of 7 hexagonal cells with one central cell surrounded by 6 cells, where the inter-cluster interference is in absent ${ }^{1}$. System parameters are set according to [17] as follows. Cell radius is $250 \mathrm{~m}$, BS transmit power is $46 \mathrm{dBm}$, noise power at receiver is $-95 \mathrm{dBm}$, and pass loss is given by $L=35.3+37.6 * \log _{10}(d), d$ is the user-to-BS distance in meters. We assume that the small scale channels are i.i.d. Rayleigh fading channels in the simulation.

\section{A. SINR simulation}

In this subsection, we observe the relationship between the average received SINR of the 1st user in the 1st cell and the large scale SIR of users in other six cells. In each simulation, we first fix the 1st user as a cell center user (user-to-BS

\footnotetext{
${ }^{1}$ In practice, this happens when different frequencies are used for adjacent clusters.
}

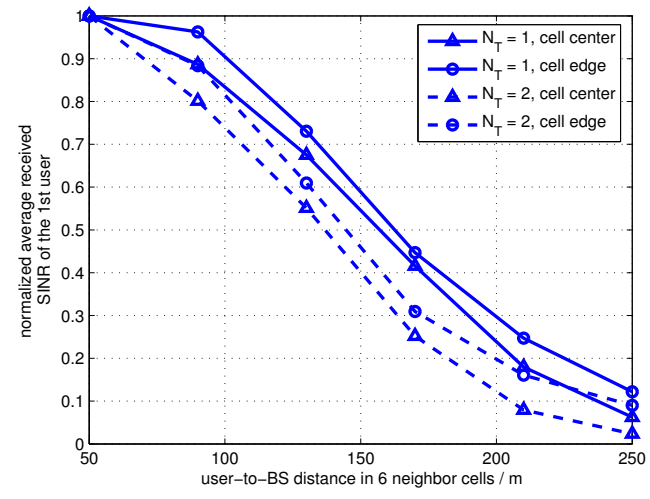

Fig. 1. SINR simulation result

distance $50 \mathrm{~m}$ ) or a cell edge user (user-to-BS distance 200m), then we adjust the user-to-BS distances in six neighbor cells to change the large scale SIR of those users and calculate the average received SINR of the 1st user correspondingly. The simulation result are averaged over 1000 Monte-carlo tests of small scale channels.

The average received SINR of the 1st user versus the userto-BS distances in six neighbor cells are shown in Fig.1. Since the SINR of the cell center user and the cell edge user differ a lot, in order to show both of them in the same figure, the SINR is normalized to make its maximum value as 1 . The curves show that the average received SINR of the 1st user decreases as the user-to-BS distance in neighbor cells grows, which indicates that the performance of the 1st user reduces as the large scale SIR of users in neighbor cells decreases. This validates our analysis using Wyner model. We can see that this conclusion is valid both in 1- and 2-antenna BS scenario and no matter if the 1st user is a cell center or a cell edge user.

\section{B. Throughput simulation}

In this subsection, we use system level simulation to evaluate the performance of our user grouping algorithm. The simulation results are averaged over 50 drops, where in each drop 40 single-antenna users are uniformly scattered in each cell. The users in 7 cells are grouped and served cooperatively by the 7 BSs using ZF precoding. Round-robin scheduling is used among the user groups. Each group of users will be served on one timeslot. Each drop ends when every user has been served once. The large scale channels are invariant during each drop, while the small scale channels vary each timeslot.

We use the cell throughput and the cell edge throughput as the performance metrics, which are denoted by $R_{\text {cell }}$ and $R_{\text {edge }}$, respectively. $R_{\text {cell }}$ is defined as the aggregate throughput of all users normalized by channel bandwidth then divided by the number of cells, while $R_{\text {edge }}$ as the $5 \%$ point of the cumulative distribution function of the user throughput normalized by channel bandwidth [18]. In our simulation, the user throughput are obtained using Shannon capacity formula.

We compare our SWG algorithm with random grouping (RG), IBG and NUS. Among these algorithms, RG do not 
use any channel information, both SWG and IBG are based on the large scale SIR, while NUS uses instantaneous channel norm information. NUS can use a threshold to tradeoff the spatial diversity and multiplexing. By setting a large value for the threshold, it can achieve a high $R_{\text {cell }}$ but low $R_{\text {edge }}$, and vice versa. In order to compare our method with NUS, we set the threshold in NUS to make its cell edge performance identical to ours, then we compare the cell throughput. NUS can achieve higher $R_{\text {edge }}$ than the results in our simulation by choosing other thresholds, but it will pay much more expense of $R_{\text {cell }}$ in that case.

The simulation results are shown in Table I. The throughput performance without BS cooperation is also shown as a baseline, which is denoted as SC in the table. SWG gain over other algorithms is defined as the throughput difference between SWG and the other algorithm normalized by the throughput of the algorithm for comparison.

TABLE I

COMPARISON OF SWG, RG, IBG AND NUS

(a) 1-antenna BS

\begin{tabular}{|c|c|c|c|c|c||c|c|c|}
\hline & SC & RG & IBG & NUS & SWG & \multicolumn{3}{c|}{ SWG gain } \\
\hline & \multicolumn{5}{|c|}{$\mathrm{bps} / \mathrm{Hz}$} & & & \\
\hline$R_{\text {edge }}$ & 0.014 & 0.065 & 0.055 & 0.07 & 0.07 & $7.69 \%$ & $27 \%$ & $0 \%$ \\
\hline$R_{\text {cell }}$ & 5.54 & 8.31 & 8.36 & 7.99 & 8.30 & $-0.1 \%$ & $-0.7 \%$ & $3.88 \%$ \\
\hline
\end{tabular}

(b) 2-antenna BS

\begin{tabular}{|c|c|c|c|c|c|c|c|c|}
\hline & $\mathrm{SC}$ & RG & IBG & NUS & SWG & \multicolumn{3}{|c|}{ SWG gain } \\
\hline & & & $\mathrm{bps} / \mathrm{Hz}$ & & & over RG & over IBG & over NUS \\
\hline$R_{\text {edge }}$ & 0.04 & 0.082 & 0.07 & 0.1 & 0.1 & $22 \%$ & $43 \%$ & $0 \%$ \\
\hline$R_{\text {cell }}$ & 6.79 & 14.05 & 14.49 & 9.95 & 14.21 & $-1.1 \%$ & $-1.9 \%$ & $42 \%$ \\
\hline
\end{tabular}

(c) 4-antenna BS

\begin{tabular}{|c|c|c|c|c|c|c|c|c|}
\hline & $\mathrm{SC}$ & RG & IBG & NUS & SWG & \multicolumn{3}{|c|}{ SWG gain } \\
\hline & \multicolumn{5}{|c|}{$\mathrm{bps} / \mathrm{Hz}$} & over RG & over IBG & over NUS \\
\hline$R_{\text {edge }}$ & 0.06 & 0.09 & 0.08 & 0.125 & 0.125 & $39 \%$ & $56 \%$ & $0 \%$ \\
\hline$R_{\text {cell }}$ & 7.93 & 23.14 & 24.42 & 11.9 & 23.06 & $-0.4 \%$ & $-5.6 \%$ & $94 \%$ \\
\hline
\end{tabular}

It is shown from the table that BS cooperation can improve both the cell throughput and the cell edge throughput, compared with the performance without BS cooperation.

Both IBG and SWG use large scale CSI. Since SWG aims at achieving fairness whereas IBG is designed for BS cooperation under constrained backhaul, it is not surprising that SWG has significant cell edge throughput gain over IBG at minor expense of cell throughput. The performance of $\mathrm{RG}$ is generally between IBG and SWG.

NUS uses more channel information than SWG, which aims at maximizing the sum rate within a time slot and achieves fairness by providing all users with equal time resources. It obtains higher cell edge throughput by reducing the multiplexing data streams, thus it pays more expense on cell throughput by using longer serving time for all users. On the other hand, SWG can serve more users in the same time-frequency resource while provide fairness via exploiting the asymmetric channel feature. Hence it can achieves higher cell throughput given the same cell edge throughput.

Due to the lack of space, we do not show the extensive simulation results, which show that similar performance can be achieved when each user has more than one antennas and
ZF-BD precoding is used, and when PBPC is considered.

\section{CONCLUSiON}

In this paper, we studied low feedback user grouping in downlink cooperative transmission systems. We showed that any user's average received SINR can be improved by grouping it with users that have stronger large scale SIR in neighbor cells. Based on this observation, we proposed a novel fair user grouping algorithm using large scale SIR, "strongweak" grouping. Simulation results validate our analysis and showed that our user grouping algorithm achieves better cell edge throughput than the existing schedulers only with slight cell throughput loss.

\section{REFERENCES}

[1] M. K. Karakayali, G. J. Foschini, and R. A. Valenzuela, "Network coordination for spectrally efficient communications in cellular systems," IEEE Wireless Commun. Mag., vol. 13, no. 4, pp. 56-61, Aug. 2006.

[2] T. Yoo and A. Goldsmith, "On the optimality of multiantenna broadcast scheduling using zero-forcing beamforming," IEEE J. Select. Areas Commun., vol. 24, no. 3, pp. 528-541, Mar. 2006.

[3] O. Somekh, O. Simeone, Y. Bar-Ness, and A. M. Haimovich, "Distributed multi-cell zero-forcing beamforming in cellular downlink channels," in Proc. GLOBECOM, Nov. 2006, pp. 1-6.

[4] S. Jing, D. N. C. Tse, J. B. Soriaga, J. Hou, J. E. Smee, and R. Padovani, "Downlink macro-diversity in cellular networks," in Proc. ISIT, June 24-29, 2007, pp. 1-5.

[5] D. Kim, "Selective relative best scheduling for best-effort downlink packet data," IEEE Trans. Wireless Commun., vol. 5, no. 6, pp. 12541259, June 2006.

[6] L. Yang, M. Kang, and M.-S. Alouini, "On the capacity-fairness tradeoff in multiuser diversity systems," IEEE Trans. Veh. Technol., vol. 56, no. 4 , pp. 1901-1907, July 2007.

[7] E. A. Jorswieck, A. Sezgin, and X. Zhang, "Framework for analysis of opportunistic schedulers: average sum rate vs. average fairness," in Proc. WiOPT, Apr. 1-3, 2008, pp. 100-105.

[8] T. Yoo, N. Jindal, and A. Goldsmith, "Multi-antenna downlink channels with limited feedback and user selection," IEEE J. Select. Areas Commun., vol. 25, no. 7, pp. 1478-1491, Sept. 2007.

[9] D. Hammarwall, M. Bengtsson, and B. Ottersten, "Utilizing the spatial information provided by channel norm feedback in SDMA systems," IEEE Trans. Signal Processing, vol. 56, no. 7, pp. 3278-3293, July 2008.

[10] S. Han, C. Yang, M. Bengtsson, and A. I. Perez-Neira, "Channel normbased user scheduling in coordinated multi-point systems," in Proc. GLOBECOM, 2009.

[11] P. Marsch and G. Fettweis, "A framework for optimizing the downlink performance of distributed antenna systems under a constrained backhual," in Proc. European Wireless Conference, Apr. 1-4, 2007.

[12] A. D. Wyner, "Shannon-theoretic approach to a Gaussian cellular multiple-access channel," IEEE Trans. Inform. Theory, vol. 40, no. 6 , pp. 1713-1727, Nov. 1994.

[13] A. Tolli, M. Codreanu, and M. Juntti, "Linear cooperative multiuser MIMO transceiver design with per BS power constraints," in Proc. ICC, June 24-28, 2007, pp. 4991-4996.

[14] S. Shi, M. Schubert, N. Vucic, and H. Boche, "MMSE optimization with per-base-station power constraints for network MIMO systems," in Proc. ICC, May 19-23, 2008, pp. 4106-4110.

[15] H. Zhang, N. B. Mehta, A. F. Molisch, J. Zhang, and H. Dai, "Asynchronous interference mitigation in cooperative base station systems," IEEE Trans. Wireless Commun., vol. 7, no. 1, pp. 155-165, Jan. 2008.

[16] H. Lutkepohl, Handbook of Matrices. Chichester, England: Wiley, 1996.

[17] "Physical layer aspects for evolved universal terrestrial radio access (UTRA)," 3rd Generation Partnership Project (3GPP), Tech. Rep. TR 25.814, 2006.

[18] "Requirements related to technical performance for imt-advanced radio interface(s)," International Telecommunication Union (ITU), Tech. Rep. Draft New Report ITU-RM.[IMT.TECH], 2008. 